\title{
In vitro culture of Plasmodium berghei-ANKA maintains infectivity of mouse erythrocytes inducing cerebral malaria
}

\author{
Ronan Jambou ${ }^{1,2^{*}}$, Fatima El-Assaad ${ }^{1}$, Valery Combes $^{1}$ and Georges E Grau
}

\begin{abstract}
Background: Infection with Plasmodium berghei is a widely used model of murine malaria and a powerful tool for reverse genetic and pathogenesis studies. However, the efficacy of in vitro reinvasion of erythrocytes is generally low, limiting in vitro studies.

Methods: Plasmodium berghei ANKA-infected blood obtained from a susceptible infected mouse was cultured in various conditions and in vitro parasitaemia was measured every day to evaluate the rate of reinvasion.

Results: High quality culture media were used and reinvasion rates were improved by vigorous orbital shaking of the flask and increasing density of the medium with gelatin.

Discussion: Using these settings, reinvasion of normal mouse erythrocytes by the parasite was obtained in vitro over two weeks with preservation of the infectivity in vivo.
\end{abstract}

\section{Background}

Plasmodium berghei is an African murine malaria parasite isolated by Vincke and Bafort in Katanga (PbNK for New York-Katanga) and in Kasapa (PbANKA for Antwerpen-Kasapa) [1,2]. During natural infection, the blood stages of the parasite undergo asynchronous development with a haploid cycle of 22 hours. Their preference for immature red blood cells (RBC) $[1,3,4]$ suggests that they belong to the "vivax group". The gametocyte production is intense, with $20 \%$ of merozoites of each asexual cycle developing into gametocytes within 24 hours in phenylhydrazine-treated mice. Despite the phylogenetic distance, the conservation of housekeeping genes and of biochemical as well as genetic processes between human and murine parasites provided the first justification for their use in malaria research. Infection of mice with Plasmodium berghei and Plasmodium yoelii established the first murine models of malaria, with early studies utilizing these models to test drug efficacy (the Peter's model).

\footnotetext{
* Correspondence: rjambou@pasteur.fr

'Vascular Immunology Unit, Department of Pathology and Bosch Institute, Sydney Medical School, The University of Sydney, NSW, Australia Full list of author information is available at the end of the article

Fullist of author information is available at the end of the article
}

The first attempt to maintain the parasite in vitro was performed by serial passages through rats and tissue culture. These passages were associated with a sharp decrease in the virulence of the parasite in vivo [5]. Early studies developed culture conditions, which permitted the combination of in vivo and in vitro experiments with the same parasites [4-6], and methods for in vitro culture of gametocytes were rapidly described. Liver stages of $P$. berghei were obtained in the HepG2 cell line, which made it possible to study prophylactic drug efficacy [7]. In vitro development of Plasmodium ookinetes was achieved later on, followed by a complete in vitro development of mosquito phases. Several strains with the same genetic background [8] have been derived from the original isolates with different sensitivity to drugs. They are used for drug testing in maturation assays, molecular studies or evaluation of vaccine efficacy. Selection of mouse strains with various degrees of sensitivity for the parasite paved the way for analysis of the pathophysiology of cerebral malaria $(\mathrm{CM})[9,10]$ and of severe anemia [11].

A new area of interest opened up with the establishment of protocols to knock-out specific genes in the parasite to investigate gene function. However, these reverse genetic studies (reviewed by [12]) are still 
limited by i) a weak transfection efficiency between $10^{-3}$ to $10^{-4}$ whereas efficiency reach $10^{-2}$ in Toxoplasma gondii), ii) the limited number of markers available to counter select transfectants iii) the mechanism of integration of foreign DNA (nearly always through homologous recombination), and iv) by the time required to select transfected parasites. Indeed the pyrimethamine treatment required to select transfectants is usually performed only 10 to 15 days after injection of the parasite into mice. Using these methods GFP fluorescent parasites were obtained, which created new areas of application. Maintaining parasites in vitro for a long period of time would allow selection of transfectants in vitro. This could pave the way for in vitro studies and facilitate infection of mice by these parasites by reducing competition between wild and mutated parasites in the mouse.

\section{Methods}

\section{Maintenance of Plasmodium berghei}

The P. berghei ANKA (PbA) strain was obtained from Josef Bafort and maintained [9] by successive infection of $\mathrm{CBA} / \mathrm{Ca}$ and $\mathrm{C} 57 \mathrm{BL} / 6$ mice. Each mouse was inoculated with $10^{6}$ IRBC intra-peritoneally [10]. For each culture condition, mice were bled 7 days post-infection (when parasitaemia in blood reached more than $8 \%$ ) and infected red blood cells (IRBC) were cultured after careful removal of leukocytes. Freezing of the IRBC in liquid nitrogen was done in Alsever's solution, as described [10]. All experiments complied with the Australian guidelines for animal research and protocol \# K20/7-2006/3/4434 was approved by the University of Sydney Animal Ethics Committee.

\section{Maintenance of in vitro culture}

Culture medium was removed daily after centrifugation of the culture at $600 \mathrm{~g}$ for $10 \mathrm{~min}$. The pellet was diluted at $2.5 \%$ haematocrit with fresh medium. Non-infected red blood cells (NRBC) were obtained from healthy mice, and were added twice a week after platelets had been carefully removed from the blood by two washes and centrifugation at $400 \mathrm{~g}$. Buffy coat was removed following Janse et al [13]. Culture was conducted in $75 \mathrm{~cm}^{2}$ flasks, flushed with gas $\left(5 \% \mathrm{CO}_{2}, 5 \% \mathrm{O}_{2}, 90 \% \mathrm{~N}_{2}\right)$ and totally sealed. Effect of storage at $4^{\circ} \mathrm{C}$ of NRBC and of IRBC was checked by keeping cells in the fridge during increasing time before seeding. Selection of mature stages of the parasite was performed on an AutoMacs (Miltenyi Biotec) using the "sensitive" procedure with IRBC diluted 1:20 in PBS. Duration of the parasite life cycle and identification of the stage of the parasite in culture were evaluated by repeated Giemsa-stained thin smears on sampled IRBC.

\section{Stability of the mouse RBC in culture}

Starting culture conditions followed those described by Mons et al and Janse et al [6,13]. To avoid mild haemolysis of IRBC in culture, tests were conducted to adapt the density of the medium and the method of shaking. Concentrations of fetal calf serum (FCS) from 5 to $20 \%$ and gelatin from 0.5 to $5 \%$ were tested. IRBC were added to RPMI1640 medium supplemented by these components and maintained at $37^{\circ} \mathrm{C}$ for five days. Medium was changed daily and haemolysis was checked optically at each step. In parallel, several ways of agitating the culture were tried, including magnetic stirring and permanent orbital shaking to minimize haemolysis.

\section{Reinvasion study}

Rate of reinvasion was calculated by daily determination of the parasitaemia in culture, using thin Giemsa-stained smears. Parasites were counted on 100 fields at $\times 1,000$ magnification. Reinvasion of newly added red blood cells by the parasites after several days of culture was experimentally confirmed by labeling of i) NRBC with a green fluorescent dye (PKH-67, MINI67-IKT, Sigma) prior to addition to the culture flask and ii) parasites with hydroethidine (red fluorescence). Parasites were labeled by incubation with hydroethidine for $30 \mathrm{~min}$ at $37^{\circ} \mathrm{C}$, two days after the addition of green-NRBC to the culture. Double-labeled IRBC were observed with a confocal microscope (Olympus FV1000).

\section{Infectivity experiment}

In vivo infectivity of the cultured parasites was evaluated by inoculating IRBC in susceptible mice (CBA/Ca or C57BL/6). After 12 days of culture $10^{6}$ IRBCs were injected intra-peritoneally [10] and parasitaemia was monitored daily on thin tail vein blood smears from day 5 post-infection onwards..

\section{Final composition of the medium}

The final composition of the medium was: RPMI1640 (3/4): DMEM-F12 (1/4); bicarbonate $32 \mathrm{mM}$, HEPES $25 \mathrm{mM}$; Albumax II 0.5\%; glucose $3 \mathrm{~g} / \mathrm{L}$; hypoxanthine $200 \mu \mathrm{M}$; calcium $2 \mathrm{mM}$; gelatin $0.1 \%$; choline $1 \mathrm{mM}$. Chemicals and dyes were purchased from Sigma, except for AlbuMAX II (Gibco n¹0021-037), DMEM/F12 (with L-glutamine, Invitrogen $n^{\circ} 11330$ ), hydroethidine (Polysciences $n^{\circ} 17084$ ) and culture flasks ( $75 \mathrm{~cm}^{2}$, Corning). Cultures were maintained at $32^{\circ} \mathrm{C}$ and medium was changed daily. Fresh mouse NRBCs were added twice a week (dilution 1:4). Haematocrit was maintained at $2.5 \%$. Culture flasks were maintained closed and vertical, filled with no more than $30 \mathrm{~mL}$ and gased with a $5 \% \mathrm{CO}_{2}, 5 \% \mathrm{O}_{2}, 90 \% \mathrm{~N}_{2}$ gas mixture. Permanent shaking at $100 \mathrm{rpm}$ on an orbital shaker was applied.

\section{Results}

\section{Stability of red blood cells}

The stability of mouse RBC in culture was the first parameter that needed improving. As previously described, 
mouse IRBC maintained in culture at $37^{\circ} \mathrm{C}$ [14] presented mild haemolysis. During this study addition of $20 \%$ FCS to the medium significantly reduced this haemolysis, but consequently decreased maturation rate of the parasites after several days of culture. Haemolysis was totally abolished by addition of gelatin. Along the same line, permanent orbital shaking of the flask at 100 rpm achieved substantial mechanical disruption of the late schizonts (Figure 1) without inducing red cell alterations as seen with the magnetic stirrer. Using this setting, large numbers of well-separated merozoites were observed in culture (Figure 1A2) and the position of the merozoites on the $\mathrm{RBC}$ as well as merozoites inside $\mathrm{RBC}$ was readily detected (Figure 1A2).

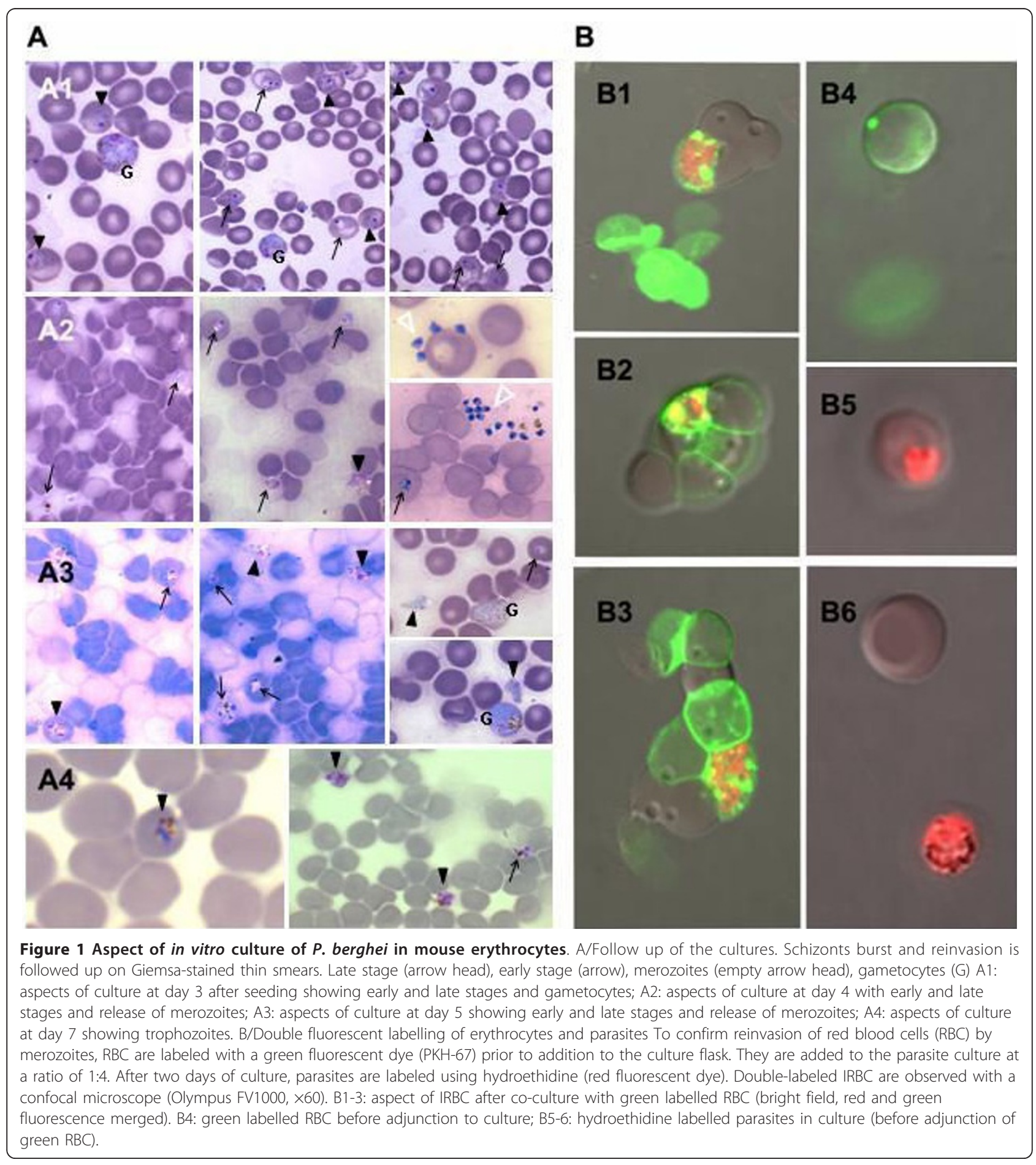




\section{Merozoite production and reinvasion}

In RPMI1640 medium supplemented in the same way as for Plasmodium falciparum [15], a decrease in the number of merozoites produced by parasites, i.e. the number of nuclei in schizonts, was observed after three life cycles of the parasite. This suggested insufficiency in nutrient levels, which was not overcome by increasing FCS concentration to more than 5\% [13]. Medium composition was modified by addition of DMEM/F12 to RPMI1640. This medium is also compatible with endothelial cell culture. Based on the follow up of in vitro parasitaemia, numerous experiments were conducted, allowing optimization of the culture medium mixture to one part DMEM/F12 and three parts RPMI1640. Higher proportion of DMEM/F12 induced a reduction of the parasitaemia. Similarly, ionic concentration in the medium was adapted in agreement with the mouse serum concentrations [16]. Addition of calcium improved their maintenance in culture. Due to a high rate of parasite multiplication and acidification of the medium, the bicarbonate concentration was increased and culture media was changed daily. The temperature was also decreased to $32^{\circ} \mathrm{C}$ without apparent change in the duration of the life cycle of the parasite. Reinvasion of newly added red blood cell by the parasites was confirmed by dual labeling of NRBC and parasites with hydroethidine (Figure 1B).

After seeding in culture, parasitaemia could be maintained between 5 to $15 \%$ for 5 to 7 days (Figure 2) with fresh blood being added twice a week. After 12 days most of the cultures had still 0.5 to $1 \%$ parasitaemia despite a dilution of the IRBC 1:4 with NRBC twice a week. Storage of the normal $\mathrm{RBC}$ at $4^{\circ} \mathrm{C}$ for up to one week before use in culture had no effect on in vitro invasion. In contrast, storage of the IRBC at $4^{\circ} \mathrm{C}$, either before seeding or after a couple of days of culture, had a dramatic effect on reinvasion (Figure 2) as already described for merozoites [17]. In most flasks, gametocyte production was rapidly observed and associated with a sharp decrease of the asexual parasitaemia (Figure 2A).

Selection of mature stage of the parasite, from infected blood was easily obtained using the AutoMacs in the same conditions as those used for P. falciparum. Following separation, parasitaemia could reach up to $90 \%$.

\section{Infectivity}

Infectivity of the cultured parasites for the host was assessed by re-injecting IRBC in mice either directly

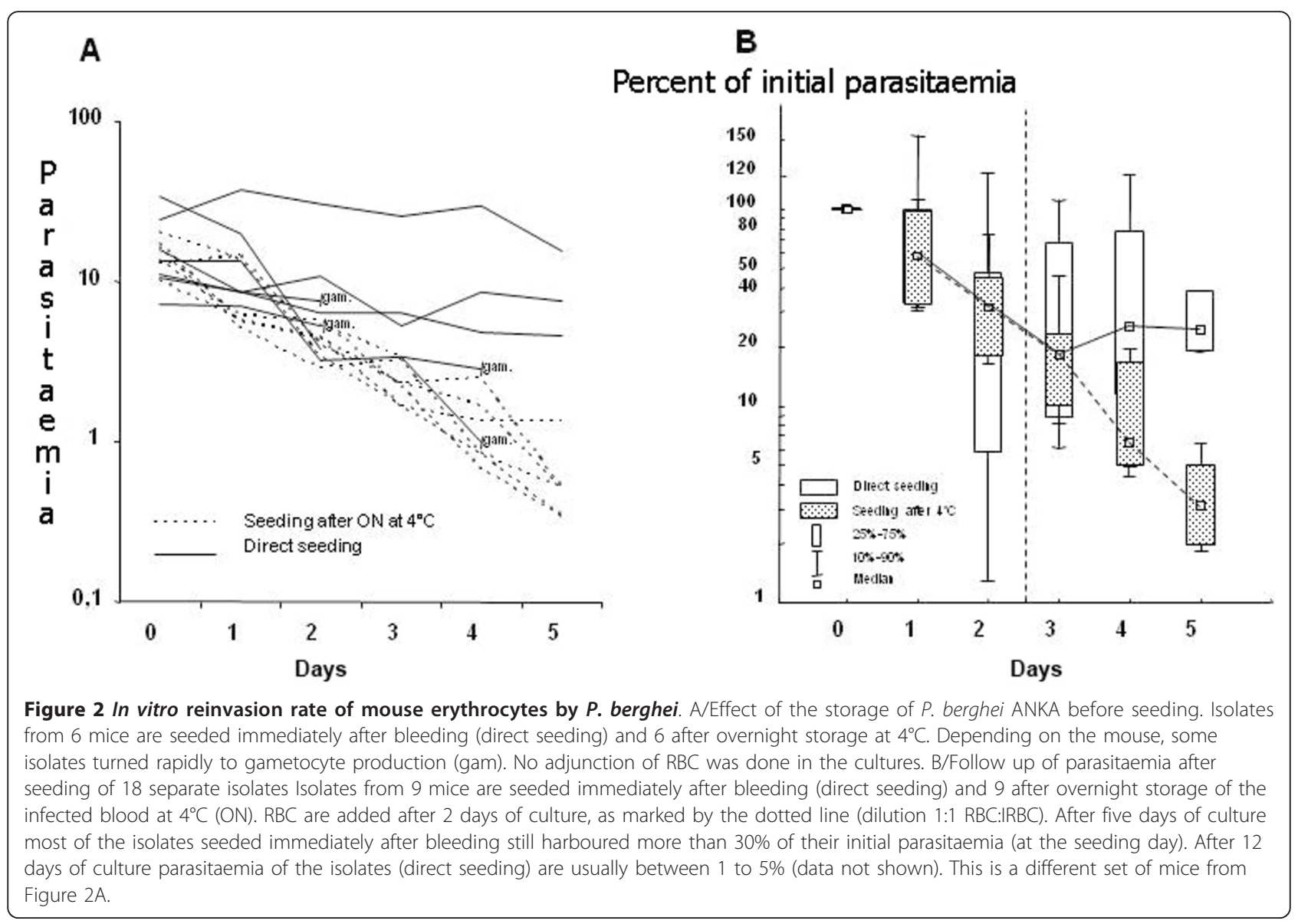


from cultures or after freezing cultured parasites in liquid nitrogen. Repeated cycles of in vitro culture of $\mathrm{PbA}$ did not affect the infectivity of the parasite for mice. After 12 days of culture, injection of $10^{6}$ IRBC in susceptible CBA/J mice readily induced $2 \%$ to $6 \%$ of parasitaemia within 7 days. Mice with high parasitaemia displayed clear signs of CM.

\section{Discussion}

In most protocols, early culture conditions for $P$. berghei closely resembled those of $P$. falciparum [13], as the maturation of young blood stages into schizonts is straightforward [18]. Long-term in vitro cultivation of $P$. berghei was claimed to be obtained by Ramaiya et al [18] at $27^{\circ} \mathrm{C}$, and by Smalley [14] who achieved a low multiplication rate at $15^{\circ} \mathrm{C}$. However, at $37^{\circ} \mathrm{C}$, a decrease in parasite density was always observed as a consequence of the instability of the red blood cells (RBC). During all these studies, re-invasion appeared to be the bottleneck of the long-term cultivation of $P$. berghei. This was attributed to two main points: i) a restricted ability to invade mature $\mathrm{RBC}$, which required adding blood with a high percentage of reticulocytes to the cultures, and ii) release of merozoites staying packed together or surrounded by RBC membrane preventing them from invading new cells. Schizonts can indeed survive for more than 20 hours in culture and most of the authors used a magnetic stirrer to increase the disruption of the IRBC. During this study, conditions of culture were adjusted to allow an average of $10 \%$ in vitro parasitaemia during the first week after seeding and at least 0.5 to $1 \%$ after 10 days. Mature stages of the parasite were easily purified using the AutoMacs procedure.

Preference of $P$. berghei for mouse reticulocytes has been widely reported and in "CM resistant" mice parasitaemia can reach $80 \%$ of $R B C[10,11]$ supposed to be mostly reticulocytes. This has implications for in vitro studies of drug sensitivity or cell/cell interactions. In this study, mice used to provide NRBC for culture were not treated before bleeding to increase reticulocytosis. Nevertheless their blood allowed regular reinvasion of $\mathrm{RBC}$ by parasites. This protocol for culture of PbAIRBC in vitro provides significant advantages particularly in allowing in vitro studies of co-culture with endothelial cells or leukocytes in conditions similar to human malaria. Cultivation of the parasites in this setting does not induce any loss of infectivity for mice.

\section{Acknowledgements and Funding}

This work was supported by funds from the National Health and Medical Research Council of Australia (NHMRC Project Grant 464893), the Australian Research Council (DP0774425) and the Rebecca L. Cooper Medical Research Foundation, Sydney, Australia. The authors also declare that they do not have a commercial or other association that might pose a conflict of interest.

\section{Author details}

"Vascular Immunology Unit, Department of Pathology and Bosch Institute, Sydney Medical School, The University of Sydney, NSW, Australia. ${ }^{2}$ Institut Pasteur de Madagascar, BP1274 Antananarivo 101, Antananarivo, Madagascar.

\section{Authors' contributions}

RJ designed the study and performed all the adaptation of the in vitro system and the confocal analysis; FEA and VC performed the in vivo studies; GEG provided support for the study, the scientific background on the Plasmodium berghei ANKA model and helped in the writing of the paper. All authors read and approved the final manuscript.

\section{Competing interests}

The authors declare that they have no competing interests.

Received: 29 October 2011 Accepted: 25 November 2011

Published: 25 November 2011

\section{References}

1. Vincke LH, Lips H: Ann Soc Belg Med Trop 1948, 28:97-104.

2. Vincke $L H$, Bafort F: Results of 2 years of observation of the cyclical transmission of Plasmodium berghei. Ann Soc Belges Med Trop Parasitol Mycol 1968, 48:439-454.

3. Gaillard G: Concours Medical 1949, 71:2395.

4. Garnham PC: The structure of early sporogonic stages of Plasmodium berghei. Ann Soc Belges Med Trop Parasitol Mycol 1965, 45:259-264.

5. Weiss ML, Degiusti DL: Modification of a malaria parasite (Plasmodium berghei) following passage through tissue culture. Nature 1964, 201:731-732.

6. Mons B, Janse CJ, Boorsma EG, Van der Kaay HJ: Synchronized erythrocytic schizogony and gametocytogenesis of Plasmodium berghei in vivo and in vitro. Parasitology 1985, 91:423-430.

7. Strome CP, De Santis PL, Beaudoin RL: The cultivation of the exoerythrocytic stages of Plasmodium berghei from sporozoites. Vitro 1979, 15:531-536.

8. Saul A, Prescott N, Smith F, Cheng Q, Walliker D: Evidence of crosscontamination among laboratory lines of Plasmodium berghei. Mol Biochem Parasitol 1997, 84:143-7.

9. Rest JR: Cerebral malaria in inbred mice, a new model and its pathology. Trans R Soc Trop Med Hyg 1982, 76:410-415.

10. Grau GE, Piguet PF, Engers HD, Louis JA, Vassalli P, Lambert PH: L3T4+ T lymphocytes play a major role in the pathogenesis of murine cerebral malaria. J Immunol 1986, 137:2348-2354.

11. Evans KJ, Hansen DS, van Rooijen N, Buckingham LA, Schofield L: Severe malarial anemia of low parasite burden in rodent models results from accelerated clearance of uninfected erythrocytes. Blood 2006, 107:1192-1199.

12. Janse CJ, Ramesar J, Waters AP: High-efficiency transfection and drug selection of genetically transformed blood stages of the rodent malaria parasite Plasmodium berghei. Nat Protoc 2006, 1:346-356.

13. Janse CJ, Mons B, Croon JJ, van der Kaay HJ: Long-term in vitro cultures of Plasmodium berghei and preliminary observations on gametocytogenesis. Int J Parasitol 1984, 14:317-320.

14. Smalley ME, Butcher GA: The in vitro culture of the blood stages of Plasmodium berghei. Int J Parasitol 1975, 5:131-132.

15. Trager W, Jensen JB: Human malaria parasites in continuous culture. Science 1976, 193:673-5.

16. Mouse Phenotype data base. [http://phenome.jax.org].

17. McAlister RO: Time-dependent loss of invasive ability of Plasmodium berghei merozoites in vitro. J Parasitol 1977, 63:455-463.

18. Ramaiya ML, Kamath VR, Renapurkar DM: Long-term in vitro cultivation of Plasmodium berghei. Int J Parasitol 1987, 17:1329-1331.

\section{doi:10.1186/1475-2875-10-346}

Cite this article as: Jambou et al: In vitro culture of Plasmodium bergheiANKA maintains infectivity of mouse erythrocytes inducing cerebral malaria. Malaria Journal 2011 10:346. 